\title{
SAG-TEMPERATURE CALCULATIONS OF TRANSMISSION LINES ON STEEP GRADES.*
}

BY

\author{
ALFRED STILL, \\ Purdue University, Lafayette, Indiana.
}

THE men engaged in the erection of overhead electric power transmission lines should be provided with tables or charts giving the sag or tension at different temperatures for a given length of span. This enables them to string the wires-either by sighting from pole to pole when erecting to the required sag, or by using a dynamometer when erecting to the required tension-so that the stress shall not exceed the specified limit under the weather conditions which will produce the assumed maximum loading.

If the storm conditions are such as to produce in the wire a stress $n$ times as great as the stress when the wire hangs in still air at the same temperature under the influence of gravity only, there will be a reduction in length due to elastic contraction when this extra load is removed, and the maximum deflection from the straight line joining the points of support will now be less than under storm conditions. It should, however, be observed that, with a sufficient rise of temperature, the elongation of the wire will be such as to produce the same maximum deflection notwithstanding a reduction in the tension. This particular temperature - which may be called the " critical" temperature-is very easily calculated, ${ }^{1}$ and since the relation between sag and tension is expressed by the well-known formula,

$$
s=\frac{w_{L}^{2}}{8 P}
$$

it follows that, if $s$ (the sag) and $l$ (the span) remain constant, we obtain the relation

or

$$
\begin{aligned}
& \frac{w}{P}=\frac{w}{P_{c}} \\
& P_{c}=\frac{P}{n}
\end{aligned}
$$

* Communicated by the Author.

${ }^{1}$ This calculation, together with formulæ for use in obtaining sag-temperature curves, are given in the writer's book, "Overhead Electric Power Transmission." 
where $n w$ is the loading per foot of wire producing the maximum tension $P$, and $w$ is the loading in pounds per foot which produces the tension $P_{o}$ when the temperature is such that the sag of the conductor hanging in still air is the same as the maximum deflection under storm conditions.

The sag at any other temperature will depend upon the length of the wire in the span, the connection between these quantities being given by the formula

$$
\lambda=l+\frac{8 s^{2}}{3 l}
$$

which is the approximate expression for the length of a parabolic arc.

When the points of support $A$ and $B$ (see Fig. I) are not on FIG. I.

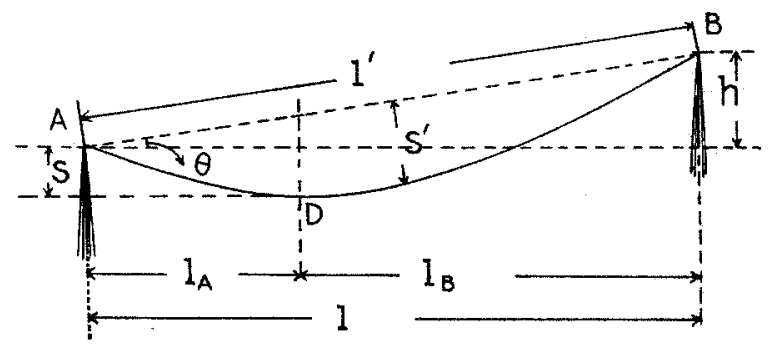

the same level, but are so nearly at the same elevation that the lowest point $(D)$ of the span lies somewhere between the two points of support, the length of wire may be considered as the sum of two half spans of lengths $l_{A}$ and $l_{B}$ respectively: this leads to the formula

$$
\lambda=l+\frac{2}{3}\left[\frac{(s+h)^{2}}{l-l_{A}}+\frac{s^{2}}{l_{A}}\right]
$$

wherein the symbols correspond to the lettering of Fig. I. By assuming different values of sag, $s$, the corresponding tension in the wire may be calculated by substituting $2 l_{A}$ for $l$ in formula (I), and the length of arc may be calculated by formula (4). Variations in the length of wire are due $(a)$ to change of tension, $(b)$ to change of temperature. The change in length due to cause $(a)$ is known, because, for a given sag, the tension is known, and it follows that the change in temperature to produce such elongation or contraction as 
is not accounted for by change of tension can easily be calculated. This outline of the method of obtaining data for the plotting of sagtemperature curves will suffice to show that small errors in calculating the length of the wire may lead to serious errors in the final results.

The horizontal distance $l_{A}$ from the lower support $A$ to the point $D$ where the wire is horizontal (see Fig. I) is readily calculated by considering that at the point $D$, where the horizontal component of the tension is $P_{k}$, the vertical component is zero;

FIG. 2.

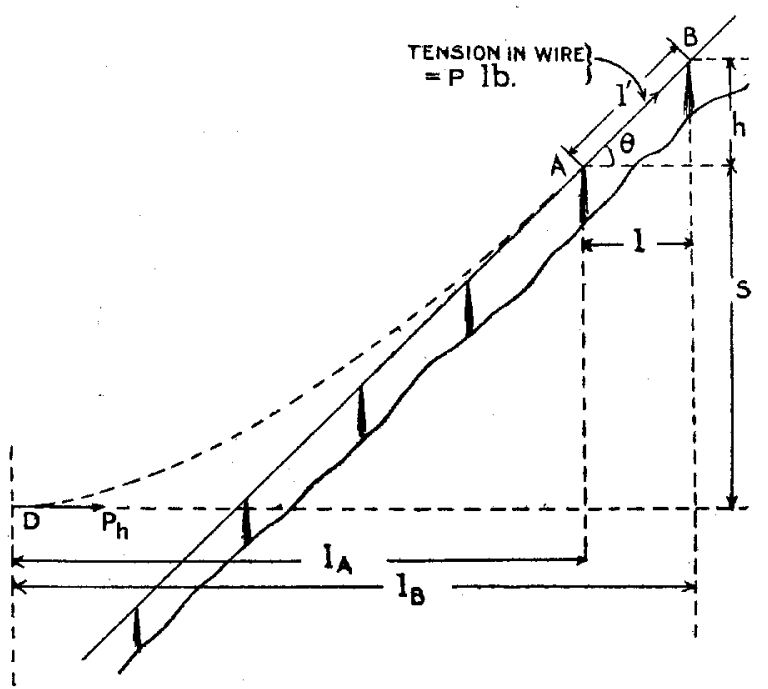

while at a point half way between $A$ and $B$ the vertical component of the tension is $P_{h} \tan \theta$, or $P_{h} \times h / l$. This must be equal to the weight of a piece of wire of which the length is $\left(\frac{l}{2}-l_{A}\right) \frac{l^{\prime}}{l}$ approximately. Thus

whence

$$
P_{h} \frac{h}{l}=w\left(\frac{l}{2}-l_{A}\right) \frac{l^{\prime}}{l}
$$

$$
l_{A}=\frac{l}{2}-\frac{h P_{h}}{w l^{\prime}}
$$

Consider now Fig. 2, where the span $A B$ is supposed to be on a steep incline. If the formula (5) is used for calculating the distance $l_{A}$ from the lower support $A$ to the point where the wire becomes horizontal, this quantity will be negative. It will determine the shape and position of an imaginary parabolic curve 
as indicated by the dotted line of Fig. 2. The vertical component of the tension at the point $B$ will be equal to the weight of the wire in the parabolic arc $B D$; while the vertical component at $A$ will be the weight of the portion $A D$. The horizontal component $\left(P_{h}\right)$ of the tension will be $P \cos \theta$, where $P$ stands for the tension in the wire at the middle of the span $A B$.

Temperature-stress calculation can be made by considering the length of the wire in the span $A B$ to be the difference between the imaginary half spans $B D$ and $A D$, the procedure being exactly as indicated above. This method is, however, objectionable, because the required lengths are very small differences between comparatively large quantities, and unless several additional terms are

Fig. 3 .

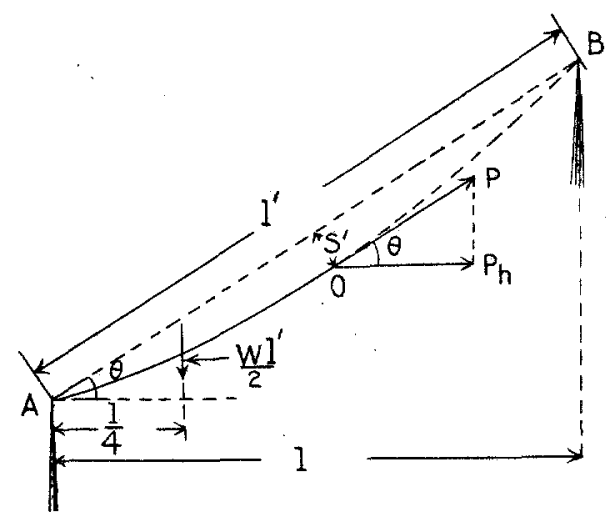

added to the approximate formula for the length of the parabolic curve, serious errors may be introduced.

The alternative method about to be explained makes use of what may be thought of as an equivalent horizontal span.

Fig. 3 is an enlarged view of the span $A B$ of Fig. 2. The deflection $s^{\prime}$ at the centre of the span may be calculated by taking moments about the point $A$. The pull $P$ in the direction $A B$, acting at a distance $s^{\prime}$, is balanced by the weight $\left(I / 2 w l^{\prime}\right)$ of the half span $A O$, acting vertically downward at an average distance $\frac{l}{4}$, whence

and

$$
P s^{\prime}=\frac{w l^{\prime}}{2} \times \frac{l}{4}
$$

$$
s^{\prime}=\frac{w l^{2}}{8 P \cos }
$$


The maximum deflection $s^{\prime}$ of the wire from the straight line $A B$ joining the points of support is always small relatively to the distance $l^{\prime}=A B$; and in computing the length of a curve which approximates to a straight line, no appreciable difference will be observable, whether the curve be considered as part of a parabola, or catenary, or ellipse, or circle. For our purpose, it will be most convenient to calculate the length of the wire by using formula (3), merely substituting $s^{\prime}$ for $s$, and $l^{\prime}$ for $l$.

The method of procedure here recommended for the calculation of sag-temperature or tension-temperature data can therefore be summed up as follows: Consider the span of either Fig. I or Fig. 3 to be replaced by an equivalent horizontal span of length $l$, being the straight line distance between points of support, and of sag $s^{\prime}$ calculated by formula (6). Consider also that the vertical loading of this imaginary span is now $w \cos \theta$ pounds per foot run, distributed uniformly over the length $l^{\prime}$, and then proceed with the calculations exactly as in the case of spans having the two supports on the same level.

When the difference in levels is considerable, as in Fig. 3, the proper correction must be made in calculating the factor $n$. Thus, if $w$ is the weight per foot of the wire (ice loading neglected) and $p$ is the maximum (horizontal) wind pressure per foot of wire, the resultant load under storm conditions will be $\sqrt{w^{2}+p^{2}}$, and the value of $n$ as previously defined will be

$$
n=\frac{V \overline{w^{2}+p^{2}}}{w}
$$

for horizontal spans. When the angle of slope, $\theta$, becomes so large that $\cos \theta$ differs appreciably from unity, we must write

$$
n=\frac{\sqrt{(w \cos \theta)^{2}+p^{2}}}{w \cos \theta}
$$

which is the value to be used in formula (2) when calculating the "critical" tension $P_{c}$ from the assumed or specified permissible maximum tension $P$.

Space does not permit of the complete working out of a numerical example; but the results of some calculations on a practical line have been plotted in Fig. 4.

The assumptions as to distance between supports, maximum load, etc., are such as would be normal-even if unreasonable-in England, where the regulations governing factors of safety and 
construction details are not such as to encourage the development of power transmission by overhead conductors. The reason for choosing data based on British rather than on American practice will be explained later.

FIG. 4 .

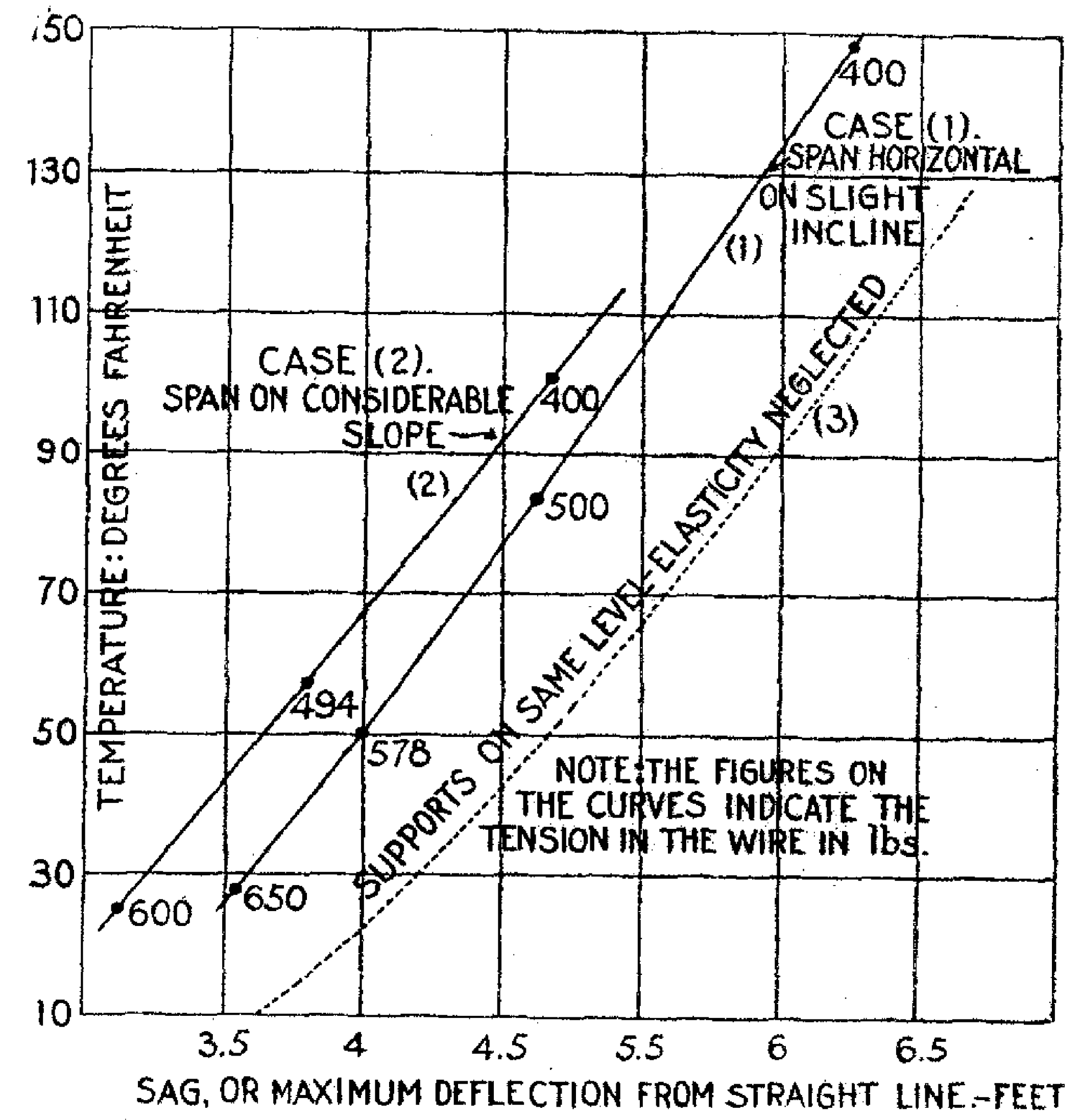

Data for calculation of results given in Fig. 4:

Wire: No. o B. \& S. solid copper. (This is almost exactly the same size as No. o S. W. G.)

Diameter of wire, $d=0.325$ in.

Cross-sectional area of wire, $A=0.083$ sq. in.

Weight of wire per foot, $w=0.3^{2} \mathrm{lb}$.

Distance between supports measured horizontally, $l=\mathrm{I} 95 \mathrm{ft}$.

Difference of elevation between points of support, $h=\mathrm{r} 40 \mathrm{ft}$.

whence, Distance between supports measured on slope, $l^{\prime}=240 \mathrm{ft}$.

Breaking stress (say) $55,000 \mathrm{lb}$. per sq. in.

Factor of safety $=5$. 
Maximum wind pressure $=I_{5} \mathrm{lb}$. per sq. $\mathrm{ft}$. of projected area of wire.

Temperature at which maximum wind pressure occurs, $t=22^{\circ} \mathrm{F}$.

The curve marked (2) in Fig. 4 gives the relation between temperature and deflection $\left(s^{\prime}\right)$ which can be measured by sighting from pole to pole on the incline. The figures on the curve are the tensions corresponding to particular temperatures. Tensions for intermediate points can very quickly be calculated by means of formula (6) if it is desired to erect the conductors with a dynamometer rather than by measuring the dip.

The curve marked (I) refers to the same span placed horizontally; that is to say, with the points of support 240 feet apart on the same level. In both cases the specified limiting tension in the wire will be reached at a temperature of $22^{\circ} \mathrm{F}$., with maximum wind-pressure in a direction perpendicular to the vertical plane passing through the points of support.

The dotted curve marked ( 3 ) in Fig. 4 is plotted from the same data as curve ( $\mathrm{r}$ ), except that the effect due to the elasticity of the wire has been neglected. The omission is often permissible, especially on long spans of aluminum wire; but on comparatively short spans of copper wire, as in the present instance, it is seen to give inaccurate results. The point has been referred to because of the peculiarity that in England-where the stringent regulations involving large factors of safety necessitate uneconomical construction with short spans-it is by no means uncommon to neglect the elasticity of the wire; while in America-where the longer spans are the cause of reduced stress variations-it is customary to take account of the changes in length due to variation of load.

Methods of Gas Warfare. S. J. M. AuLd. (Journal of the Washington Academy of Sciences, vol. 8, No. 3, p. 45, February 4, I9I8.) -Not so much is heard of gas warfare at the present time as two years ago, when the first use of that method of attack was made on the Western Front. Gas is, however, used to a tremendous extent, and the amount that has been and is being hurled back and forth in shells and clouds is almost unbelievable. The success of a cloud gas attack depends on thorough preparation beforehand. The attackers 